Chirurg 2015 $\cdot 86: 709$

DOI 10.1007/s00104-015-0037-5

Online publiziert: 24. Juni 2015

(c) Springer-Verlag Berlin Heidelberg 2015

CrossMark
O. Strobel · M.W. Büchler

Klinik für Allgemein-, Viszeral- und Transplantationschirurgie,

Universitätsklinikum Heidelberg, Heidelberg, Deutschland

\title{
Die intraoperative Cholangiographie senkt das Risiko der Gallenwegsverletzung im Rahmen der Cholezystektomie bei und nach akuter Cholezystitis
}

\section{Originalpublikation \\ Törnqvist B, Strömberg C, Akre O et al. (2015) Selective intraoperative cholangiography and risk of bile duct injury during cholecystec- tomy. Br J Surg Apr 28 [Epub ahead of print] PMID: 25919401}

\section{Hintergrund und Fragestellung}

Gallenwegsverletzungen im Rahmen einer Cholezystektomie können schwerwiegende Folgen haben. $\mathrm{Ob}$ das Risiko für Gallenwegsverletzungen durch eine routinemäßige intraoperative Cholangiographie gesenkt werden kann und welche Patienten von einer Cholangiographie profitieren, wird kontrovers diskutiert. Törnqvist et al. haben dies anhand der „Swedish Registry for Gallstone Surgery" (GallRiks) untersucht, in der etwa $90 \%$ der in Schweden durchgeführten Cholezystektomien erfasst sind.

\section{Methoden}

Es wurden alle Cholezystektomien analysiert, die zwischen 05/2005 und 12/2010 erfasst wurden. Gallengangsverletzungen wurden definiert als jede Verletzung des Gallengangssystems außer einer intraoperativen Verletzung der Gallenblase oder des Ductus cysticus. Postoperative Gallelecks einschließlich einer Zystikusstumpfinsuffizienz wurden mit einbezogen. Patienten- und prozedurenbezogene Risikofaktoren für eine Gallengangsverletzung wurden mittels multivariater Regressionsanalyse untersucht mit einem Fokus auf die Durchführung einer intraoperativen Cholangiographie.

\section{Ergebnisse}

Bei insgesamt 51.041 Cholezystektomien traten 747 (1,5\%) Gallenwegsverletzungen auf. Von den Cholezystektomien wurden $15.462(30,3 \%)$ nicht elektiv durchgeführt. 9008 (18,6\%) der Cholezystektomien wurden bei einer akuten Cholezystitis, 5787 (11,3\%) bei Zustand nach akuter Cholezystitis durchgeführt. Eine laparoskopische Cholezystektomie erfolgte in 89,9\%. Die Konversionsrate betrug 9,0\%. Eine intraoperative Cholangiographie wurde in $44.401(87,7 \%)$ von 50.654 Operationen versucht. Von 42.436 erfolgreichen Cholangiographien (technische Erfolgsrate: 95,6\%) wurde in 5068 Fällen (12,0\%) eine Choledocholithiasis gefunden. Risikofaktoren für eine Gallenwegsverletzung waren:

- höheres Patientenalter und ASAScore,

- notfallmäßige Aufnahme,

- offene Cholezystektomie,

- Vorliegen einer akuten Cholezystitis und

- Zustand nach akuter Cholezystitis sowie

- das Vorliegen einer Choledocholithiasis.

Bei Patienten mit akuter Cholezystitis gab es keinen signifikanten Zusammenhang zwischen Zeitpunkt der Operation nach Aufnahme und dem Risiko für Gallenwegsverletzungen. Die Rate an Gallenwegsverletzungen stieg aber nach dem 5 . Tag auf über $3 \%$ an. Die intraoperative Cholangiographie war mit einem verminderten Risiko für eine Gallenwegsverletzung assoziiert (Odds Ratio [OR]: 0,76; $95 \%$-Konfidenzintervall: 0,62-0,93). Dieser Vorteil beschränkte sich aber auf $\mathrm{Pa}$ - tienten mit akuter Cholezystitis (OR: 0,44; 0,30-0,63) und Zustand nach Cholezystitis (OR: 0,$59 ; 0,35-1,00$ ).

\section{Diskussion}

Die Studie zeigt, dass die Durchführung einer intraoperativen Cholangiographie bei Patienten, die bei Vorliegen einer akuten Cholezystitis oder bei Zustand nach einer akuten Cholezystitis operiert werden, das Risiko für eine Gallenwegsverletzung senkt und daher empfehlenswert ist. Die Ergebnisse legen zwar nahe, dass die routinemäßige Cholangiographie nicht bei allen Patienten notwendig ist. Die Schlussfolgerung der Autoren, dass der Nutzen der intraoperativen Cholangiographie sich auf die akute Cholezystitis und den Zustand nach akuter Cholezystitis beschränkt, wird durch die vorliegenden Daten allerdings nicht komplett gestützt. So ist beispielsweise das Vorliegen einer Schrumpfgallenblase bei chronischer Cholezystitis sicher ebenfalls mit einem erhöhten Risiko für Gallenwegsverletzungen assoziiert, wurde aber in der Studie nicht untersucht.

\section{Korrespondenzadresse}

PD Dr. med. O. Strobel

Klinik für Allgemein-, Viszeral- und Transplantationschirurgie Universitätsklinikum Heidelberg Im Neuenheimer Feld 110, 69120 Heidelberg Oliver.Strobel@med.uni-heidelberg.de

\section{Einhaltung ethischer Richtlinien}

Interessenkonflikt. O. Strobel und M.W. Büchler geben an, dass kein Interessenkonflikt besteht. 